\title{
ANÁLISE CRÍTICA DA PESQUISA EM COMPORTAMENTO INFORMACIONAL SEGUNDO AS DEMANDAS DA EMPRESA INTENSIVA EM CONHECIMENTO
}

\author{
CRITICAL ANALYSIS OF RESEARCH IN INFORMATIONAL BEHAVIOR ACCORDING \\ TO THE DEMANDS OF THE INTENSIVE KNOWLEDGE COMPANY
}

\author{
José Osvaldo De Sordi ${ }^{1}$ \\ Marcia Carvalho de Azevedo ${ }^{2}$ \\ Manuel Meireles ${ }^{3}$
}

\begin{abstract}
RESUMO
Empresas com uso intensivo do recurso conhecimento (KIF) demandam processo de gestão da informação abrangente e complexo em termos de atividades. Os modelos de comportamento informacionais utilizados nas pesquisas em gestão da informação são originários da Ciência da Informação e voltam-se para interesses pontuais das bibliotecas, concentram-se em atividades de busca e seleção da informação. Estes modelos não abrangem, por exemplo, comportamentos atrelados às atividades de aprendizagem, contribuição e descarte da informação. Esta pesquisa volta-se a esta lacuna, ou seja, analisa a adequação das pesquisas e modelos científicos em comportamento informacional em relação à diversidade de comportamentos informacionais requeridos aos trabalhadores que atuam nas KIF. Observou-se a carência de pesquisas científicas que analisem os comportamentos informacionais de forma ampla, diversificada e integrada, segundo a demanda das KIF. Além de evidenciar essa lacuna/oportunidade para pesquisas científicas, apresenta-se um quadro abrangente e consolidador dos diversos comportamentos informacionais encontrados dispersos na literatura, classificados segundo cada uma das diversas etapas do processo de gestão da informação demandado pelas KIF.
\end{abstract}

PALAVRAS-CHAVE: Comportamento informacional. Gestão da informação. Trabalhador da informação. Trabalhador do conhecimento.

\begin{abstract}
Firms with intensive use of the knowledge resource (KIF) demand a comprehensive and complex process of information management in terms of activities. The informational behavior models used in information management research are originated in Information Science and are directed to specific interests of libraries, focused on activities of search and selection of information. These models do not cover, for example, behaviors related to learning activities, contribution and disposal of information. This research addresses this gap, it analyses the adequacy of the research and scientific models in information behavior in relation to the diversity of informational behaviors required to employees who work in KIF. There was a lack of scientific research that analyzes the informational behavior in a large, diversified and integrated manner, according to the demand of KIF. Besides showing this gap/opportunity for scientific research, this paper presents a comprehensive and consolidated framework of the various informational behaviors found scattered in the literature, classified according to each of the different stages of the information management process demanded by KIF.
\end{abstract}

KEYWORDS: Informational behavior. Information management. Information worker. Knowledge worker.

\footnotetext{
${ }^{1}$ Docente-pesquisador do Programa de Mestrado em Administração do Centro Universitário das Faculdades Metropolitanas Unidas (FMU). Docente-pesquisador colaborador do Programa de Mestrado Profissional em Administração da FACCAMP. E-mail: osdesordi@gmail.com

${ }^{2}$ Professora e coordenadora do curso de administração da Universidade Federal de São Paulo. Atua no curso de graduação e mestrado em Administração. E-mail: marciacarvalhoazevedo@gmail.com

${ }_{3}^{3}$ Coordenador, pesquisador e professor do programa de mestrado profissional em administração da FACCAMPInstituto Campo Limpo Paulista e professor conteudista da UNIP. E-mail: profmeireles@uol.com.br

Recebido em: 15/07/2015 - Aceito em: 19/08/2015
} 


\section{INTRODUÇÃO}

As pesquisas científicas em comportamento informacional concentram-se em algumas atividades, sendo a seleção de informação a mais amplamente estudada (CASE, 2002). A definição de comportamento informacional de Wilson (1999) é a mais difundida na academia, ela é proveniente da área de Ciência da Informação, e destaca três atividades: identificação da necessidade, busca e uso da informação. No contexto específico da área da Ciência Gerencial, especificamente da administração da informação, Choo (2011) aborda o mesmo conjunto de comportamentos, para o mesmo conjunto específico de atividades. Este cenário, de pesquisas de comportamento informacional centradas em algumas poucas atividades, mostra-se limitado em comparação com as diversas atividades requeridas pelas atuais abordagens para gestão da informação nas organizações (MISHRA, BHASKAR, 2011; BUKOWITZ, WILLIAMS, 1999). A atual sociedade da informação apresenta a informação como o principal recurso organizacional (GOREY; DOBAT, 1996), requerendo uma visão holística e integrada desse recurso (SVEIBY, 2001).

Spink (2004) observou a pouca sinergia e integração dos modelos de comportamento informacional disponíveis na academia. Destacou o distanciamento desses modelos da realidade atual dos profissionais da sociedade da informação, que se engajam em múltiplas atividades envolvendo a informação, demandando múltiplos comportamentos informacionais, simultaneamente, do mesmo profissional. Esta dificuldade é mais perceptível nas organizações que fazem uso intensivo do recurso conhecimento, denominadas knowledge intensive firms (KIF), descritas no capítulo seguinte. As KIF requerem de seus funcionários um conjunto mais extenso e complexo de atividades associadas às informações, que vão além da busca e uso de informações. A realização desse conjunto amplo de atividades é fundamental para que essas empresas possam operacionalizar e articular a espiral da gestão do conhecimento, ou seja, estabelecer um ciclo virtuoso de conhecimento gerando conhecimento (NONAKA; TAKEUCHI, 1995). Essas organizações demandam, por exemplo, comportamentos informacionais associados às atividades de criação, contribuição e descarte de conteúdo, muitas vezes requeridos de um mesmo profissional, o knowledge worker, também definido no próximo capítulo.

Dentro desse contexto se define o objetivo e o problema da presente pesquisa. O problema caracteriza-se pela aparente pouca abrangência das pesquisas em comportamento informacional na investigação da diversidade de comportamentos requeridos para operacionalização plena e eficiente das diversas atividades associadas ao processo de gestão da informação das KIF. Suposição concebida pela observação do escopo mais restrito dos processos realizados nas bibliotecas, os quais fundamentaram os modelos e conceitos de comportamento informacional empregados nas pesquisas contemporâneas em gestão da informação nas organizações. Desta forma, tem-se como objetivo desta pesquisa: analisar as pesquisas científicas, publicadas em periódicos científicos, com foco em comportamento informacional, verificando a diversidade, conceituação e integração de comportamentos associados às demandas do processo de gestão da informação no contexto das organizações. 
Para justificar o tema comportamento informacional no contexto da KIF, observou-se que as pesquisas nesta área encontram-se centralizadas em torno de algumas poucas atividades do processo de gestão da informação (MISHRA; BHASKAR, 2011). Foi constatada também a pouca sinergia e integração dos modelos de comportamento informacional disponíveis na academia (SPINK, 2004). Para evidenciar a relevância do tema, destaca-se que os comportamentos informacionais dominantes, denominados de cultura da informação, são operacionalizados por intermédio de valores, normas e práticas organizacionais, com impacto direto na forma como a informação é percebida, criada e utilizada (CHOO et al., 2008). Aos praticantes da área de gestão, que atuam no contexto da KIF, a identificação e descrição de subgrupos específicos de comportamentos informacionais, conforme identificados e descritos ao longo desta pesquisa, constituem-se em insumo útil não apenas para análise dos comportamentos informacionais, mas, também, para as atividades de planejamento, discussão e definição das normas, práticas e valores, que deverão balizar e conduzir as ações dos trabalhadores da KIF aos comportamentos informacionais esperados.

\section{REFERENCIAL TEÓRICO}

A maior parte do trabalho realizado nas knowledge intensive firms (KIF) é de natureza intelectual e os funcionários qualificados constituem grande parte da força de trabalho (ALVESSON, 2000). Os trabalhadores que atuam nas KIF, além de serem dotados de grande capacidade intelectual, apresentam vocação empreendedora (STARBUCK, 1992). As KIF enfatizam os investimentos em capital intelectual e seus membros compartilham da percepção de ser a gestão do conhecimento uma prática crucial ao estabelecimento de vantagem competitiva (ALVESSON, 2011). Nas KIF há incentivo organizacional para o livre trâmite de fluxos de conhecimentos e expertise entre os setores da empresa. Por serem entidades relativamente novas, não há modelos ou métodos organizacionais voltados para KIF. Presume-se que elas sejam organizadas e pautadas por operações adhocráticas, estruturas e processos flexíveis e com alto índice de inovação (STARBUCK, 1992). As operações adhocráticas são informais e adaptáveis, uma forma oposta ao que ocorre na burocracia (DOLAN, 2010).

Um ator muito importante no contexto das KIF são os trabalhadores do conhecimento ou knowledge worker (KW). Segundo Frenkel et al. (1995), o KW é o profissional que: (a) tem alto nível de criatividade em seu trabalho, (b) faz uso extensivo de capacidades intelectivas, e (c) faz mais uso de conhecimento teórico do que conhecimento contextual. $\mathrm{O}$ KW difere-se do trabalhador da informação, ou information worker (IW), principalmente pela capacidade de trabalhar e aplicar conhecimento teórico para geração de novos conhecimentos, enquanto que o IW volta-se ao conhecimento contextual, ou seja, a aplicação em diferentes contextos do que já é conhecido. Segundo Hislop (2008) esta é uma característica muito importante de ser observada e destacada, considerando os frequentes equívocos dos que incluem as profissões clássicas, como arquiteto, médicos, engenheiro de software, advogado, consultores, contadores, como KW. Estes criam novos produtos e serviços a partir do domínio de conceitos e técnicas previamente adquiridos, ou seja, 
conhecimento contextual das suas profissões. Não se cria uma nova informação (conhecimento), o que se faz é aplicar conhecimentos prévios, adquiridos em sua formação, para geração de um novo produto ou serviço no contexto da sua profissão.

Para as KIF um recurso crítico ao sucesso da organização é a eficácia do seu processo de gestão da informação e, principalmente, das atividades de criação/desenvolvimento, elevando-o para um estágio do processo de gestão do conhecimento. Mishra e Bhaskar (2011) realizaram uma revisão da literatura com o foco na identificação das principais atividades associadas às diferentes versões de processos apresentados sobre a denominação de gestão da informação ou de gestão do conhecimento. Trabalho similar ao desenvolvido por Lytras e Pouloudi (2003), que consolidou não apenas a perspectiva acadêmica e científica dos processos de gestão da informação e de gestão do conhecimento, mas também as versões mais pragmáticas das grandes corporações internacionais, entre elas, as versões utilizadas pelas grandes consultorias em negócios. Nesses trabalhos de revisão, observa-se um conjunto de atividades dominantes que se repetem na maioria dos processos de gestão da informação e de gestão do conhecimento, são elas: obtenção da informação, utilização, aprendizagem, criação, compartilhamento, avaliação e descarte.

Para eficácia do processo de gestão da informação ou de gestão do conhecimento nas organizações, espera-se que seus executores e gestores ajam dentro de um contexto de comportamento informacional coerente e sinérgico entre as diversas atividades do processo de gestão da informação. Por comportamento informacional entende-se (KARUNAKARAN; SPENCE; REDDY, 2010, p.2) "a totalidade de comportamentos exibidos quando do trabalho conjunto das pessoas em identificar uma necessidade de informação, recuperar, buscar e compartilhar essa informação, além de avaliar, sintetizar e dar sentido a essa informação encontrada e, em seguida, utilizar a informação encontrada". Observa-se que esta definição é mais aderente ao processo de gestão da informação, pois não aborda a criação, apenas a utilização, ou seja, captação/transferência da informação já conhecida para aplicação na sequência. Adota-se esta definição, embora não seja a mais difundida, em função das demais definições e modelos de comportamento informacional serem mais restritos e fortemente influenciados pelo modelo de Wilson (1999), ou seja, são abordagens que não priorizam a análise e utilização da informação (BARTLETT e TOMS, 2005). Observa-se que a atividade denominada de "pesquisa" (information seeking) por Wilson (1999), dentro do contexto das bibliotecas, é denominado pelos autores da área de gestão da informação como "captura", "aquisição", "busca", dentre outros (LYTRAS, POULOUDI, 2003; MISHRA, BHASKAR, 2011), sendo o termo "busca" o mais frequentemente empregado e, por esta razão, o termo utilizado nesse artigo.

\section{MÉTODO DA PESQUISA}

Para identificação de pesquisas científicas associadas ao tema comportamento informacional, descritas na forma de artigos e publicadas nas principais revistas científicas, explorou-se os repositórios de textos científicos EBSCO e ProQuest. Para esses dois 
repositórios, utilizaram-se senhas com perfil de acesso o mais amplo possível, contemplando todas as bases de dados disponíveis, abrangendo as específicas da área de gestão, respectivamente, Business Source Complete e ABI/INFORM Complete. As atividades de busca e seleção de artigos ocorreram durante os meses de dezembro de 2013 e janeiro de 2014. As pesquisas iniciais (scanning) concentraram-se no termo "information behavior" presente no título do artigo. A leitura skimming desses artigos demonstrou a existência de três grandes grupos de artigos que, acrescidos ao termo inicial, constituíram quatro grupos centrais de palavras-chave a serem utilizadas como critério de pesquisa junto ao software de busca dos dois repositórios: "information behavior", "information sharing / share", "information use / usage / using" e "information seeking".

A Quadro 2 descreve as palavras-chave utilizadas como critério de pesquisa junto aos softwares de busca dos dois repositórios. Estas palavras-chave foram associadas aos campos título e resumo do comando de pesquisa (query) e, por conseguinte, confrontadas com os atributos respectivos (metainformações) dos artigos indexados nos dois repositórios. Este procedimento resultou na identificação de 246 artigos. Destes, foram excluídos: a) os artigos idênticos, ou seja, presentes nos dois repositórios; b) as resenhas de livros; e c) as cartas de editores. Este procedimento resultou em 133 artigos, conforme descrito na última coluna da Quadro 2, ou seja, os artigos efetivamente submetidos à técnica de análise de conteúdo. Para estes artigos, procedeu-se à leitura intensiva, com o objetivo de identificar os comportamentos humanos associados ao trabalho, à lida da informação, e descritos pelos autores do artigo como comportamento informacional. Desta forma as unidades de registro resultantes e empregadas para análise de conteúdo foram: "comportamento de compartilhamento", "comportamento de busca", "comportamento de uso", "comportamento de aprendizagem" e "comportamento de obtenção". Na seção seguinte, estes comportamentos são definidos, exemplificados e discutidos, a partir de textos extraídos dos artigos da amostra. 
QUADRO 1 - Critérios de pesquisa empregados e quantidade de artigos científicos encontrados nos repositórios referentes ao tema comportamento informacional

\begin{tabular}{|c|c|c|c|c|}
\hline \multirow{2}{*}{ Grupo } & \multirow{2}{*}{ Critérios de Seleção $^{[1]}$} & \multicolumn{2}{|c|}{ Retorno da Pesquisa } & \multirow{2}{*}{$\begin{array}{l}\text { Amostra } \\
\text { \# artigos }\end{array}$} \\
\hline & & EBSCO & ProQuest & \\
\hline $\begin{array}{l}\text { Information } \\
\text { Behavior }\end{array}$ & $\begin{array}{l}\text { "information behavior" no Título } \\
\text { E "information behavior }{ }^{[3] " ~ n o ~ R e s u m o ~}\end{array}$ & 25 & 45 & 44 \\
\hline $\begin{array}{l}\text { Information } \\
\text { Seeking }\end{array}$ & $\begin{array}{l}\text { “information seeking” no Título } \\
\text { E “behavior” no Resumo }\end{array}$ & $92^{[2]}$ & 31 & 34 \\
\hline $\begin{array}{l}\text { Information } \\
\text { Sharing }\end{array}$ & $\begin{array}{l}\text { [information sharing no Título E “behavior” no Resumo] } \\
\text { + [information share no Título E “behavior” no Resumo] }\end{array}$ & 21 & 7 & 29 \\
\hline $\begin{array}{c}\text { Information } \\
\text { Use }\end{array}$ & $\begin{array}{l}\text { [“information using” no Título E “behavior” no Resumo] } \\
+ \text { [ "information use” no Título E “behavior” no Resumo] } \\
+ \text { ["information usage" no Título E “behavior” no Resumo] }\end{array}$ & 19 & 6 & 26 \\
\hline & Total de artigos: & 157 & 89 & 133 \\
\hline
\end{tabular}

[1] Selecionaram-se, também, as opções "somente artigos científicos" e "com texto completo disponível"

[2] Para redução e maior pertinência dos 92 artigos que retornaram, alterou-se o critério de pesquisa no resumo: do termo "behavior" para "information behavior", o que resultou em 10 artigos. O número total do retorno foi mantido na tabela a fim de evidenciar e apresentar uma ordem de grandeza com relação ao interesse da academia pelos temas associados a comportamento informacional

[3] A busca foi realizada com as palavras behavior e behaviour

Fonte: Elaboração dos autores

\section{ANÁLISES E DISCUSSÕES}

As primeiras cinco subseções desse capítulo descrevem a conceituação e a diversidade dos comportamentos informacionais identificados nos artigos da amostra da pesquisa. Há uma subseção para cada uma das cinco unidades de registro empregadas para análise de conteúdo: "comportamento de compartilhamento", "comportamento de busca", "comportamento de uso", "comportamento de aprendizagem" e "comportamento de obtenção". Para cada um desses comportamentos, registraram-se os subconjuntos de comportamentos informacionais descritos nas pesquisas da amostra, descritos nas subseções a seguir e resumidos na Quadro 1. Os comportamentos indicados como positivos e comportamentos indicados como negativos ao processo de gestão da informação nas organizações, também foram registrados e discriminados na Quadro 1. A última subseção deste capítulo analisa o nível de integração de comportamentos informacionais abordados nas pesquisas descritas nos artigos da amostra. 


\subsection{Sobre comportamento de compartilhamento da informação}

A comunicação pode ser mediada pelo homem, com emissor e receptor humano, ou assistida pela tecnologia, com a emissão e/ou recepção fazendo uso dos recursos de tecnologia da informação e comunicação (TIC). Nos comportamentos informacionais associados à interação humana identificou-se a comunicação no formato de voz e gesticulação. Para voz identificou-se a interlocução junto a dois grupos de pessoas: conhecidas, utilizando o comportamento informacional face-a-face (TURNER, 2012); e desconhecidas, empregando o comportamento boca-a-boca (PARIDON, 2005). Alguns comportamentos de comunicação humana apresentam denominações específicas. Eles estão relacionados a protocolos, procedimentos e propósitos de comunicação bem definidos: participar de grupo de discussão (O'CONNOR, 2013; LILJENQUIST, GALINSKY, KRAY, 2004), prover feedback (WOFFORD; CALABRO; SIMS, 1975) e prover apoio técnico (HASSAN IBRAHIM; ALLEN, 2012). O compartilhamento por gesticulação ou visualização foi identificado com a pessoa transmissora se deixando ser observada pela pessoa receptora, bem como interagindo com a prática do trabalho, ou seja, comportamento de visualização e/ou realização conjunta com a pessoa que está trabalhando, comportamento de compartilhamento pela prática do fazer (MEYER, 2003). Observaram-se alguns comportamentos de compartilhamento de informação bastante específicos em função de limitações do público receptor, por exemplo, em comunidades orais que não dominam a escrita, identificaram-se os comportamentos informacionais de: repetição da fala por duas ou três vezes e adoção de padrões mnemônicos, como expressões estereotipadas, aliterações, assonâncias, apelidos, provérbios e adivinhas (MEYER, 2003). 
QUADRO 2 - Subconjuntos de comportamentos informacionais descritos nas pesquisas da amostra

\begin{tabular}{|c|c|c|c|c|c|c|}
\hline & & \multicolumn{5}{|c|}{ Atividades da Gestão da Informação nas Organizações } \\
\hline & & Compartilhar & Buscar & Utilizar & Aprender & Obter \\
\hline 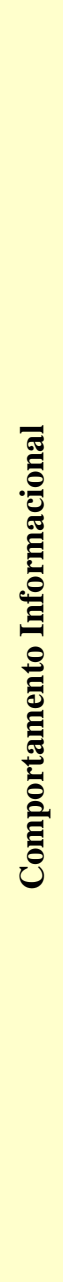 & : & $\begin{array}{l}\text { Transmissão } \\
\text { - e-mails } \\
\text { - arquivos } \\
\text { Disponibilização } \\
\text { - base de dados } \\
\text { - blog } \\
\text { Fala } \\
\text { - cara a cara } \\
\text { - boca a boca } \\
\text { - repetidas vezes } \\
\text { - usando padrões } \\
\text { mnemônicos } \\
\text { - grupo de discussão } \\
\text { - feedback } \\
\text { - apoio técnico } \\
\text { Gesticulação } \\
\text { - deixar ser observado }\end{array}$ & $\begin{array}{l}\text { Pesquisa ativa } \\
\text { - monitoramento ( pesquisa sistemática } \\
\text { no acervo) } \\
\text { - scanning (pesquisa rápida e extensa no } \\
\text { acervo a partir de metadados e } \\
\text { metainformações) } \\
\text { - quanto à fonte ser conhecida ou não } \\
\text {--- activeseeking (pesquisa em fonte } \\
\text { desconhecida) } \\
\text {--- activescanning (pesquisa em fonte } \\
\text { conhecida) } \\
\text { - quanto à quantidade de temas } \\
\text { pesquisados } \\
\text {--- multitasking (múltiplas buscas } \\
\text { simultaneamente) } \\
\text { - quanto a considerar e solicitar } \\
\text { classificação do retorno da pesquisa por } \\
\text { relevância } \\
\text {--- distinguishing (categoriza o retorno } \\
\text { por relevância segundo os critérios } \\
\text { definidos pelo próprio pesquisador) } \\
\text {--- assessing (categoriza o retorno por } \\
\text { relevância segundo os critérios pré- } \\
\text { definidos pelo software) } \\
\text { Observação passiva } \\
\text { - casual / broad scanning (navega de } \\
\text { forma descompromissada em ambientes } \\
\text { virtuais e habituais de pesquisa, pré- } \\
\text { selecionados) } \\
\text { - nondirected monitoring (encontra } \\
\text { informação útil em locais incomuns ou } \\
\text { improváveis) }\end{array}$ & $\begin{array}{l}\text { Empacotamento da } \\
\text { informação (information } \\
\text { packaging) } \\
\text { - a informação é reunida e } \\
\text { consolidada com outras, } \\
\text { compondo um novo documento } \\
\text { Desenvolvimento de novos } \\
\text { conhecimentos e insights } \\
\text { (developing new knowledge } \\
\text { and insights) } \\
\text { - a informação é utilizada em } \\
\text { um processo de orientação } \\
\text { prospectiva envolvendo } \\
\text { elementos cognitivos e afetivos } \\
\text { Direcionando julgamentos e } \\
\text { decisões (shaping judgments } \\
\text { and decisions) } \\
\text { - a informação é considerada em } \\
\text { um processo de tomada de } \\
\text { decisão } \\
\text { Influenciando outros } \\
\text { (influencing others) } \\
\text { - a informação é utilizada no } \\
\text { processo de interação } \\
\text { interpessoal para alterar } \\
\text { entendimento e comportamento } \\
\text { de outros } \\
\text { Reconhecimento da existência } \\
\text { da informação } \\
\text { - a informação passa por um } \\
\text { processo de leitura reflexiva e o } \\
\text { leitor toma ciência }\end{array}$ & $\begin{array}{l}\text { Criação coletiva de sentido } \\
\text { (sensemaking) } \\
\text { - consistentemente organizar as } \\
\text { informações em listas, tabelas e } \\
\text { gráficos } \\
\text { - desenvolver e utilizar } \\
\text { indicadores da empresa para } \\
\text { identificar problemas e soluções } \\
\text { - apreciar feedback, mesmo } \\
\text { quando negativo } \\
\text { Co-autoria } \\
\text { - desenvolver textos em rede } \\
\text { colaborativa / "assembling" } \\
\text { Síntese } \\
\text { - compilar e comparar } \\
\text { informações provenientes de } \\
\text { diferentes fontes }\end{array}$ & $\begin{array}{l}\text { Organização } \\
\text { - Criar catálogos de } \\
\text { informação } \\
\text { - Indexar a informação } \\
\text { - Manter base de dados } \\
\text { bibliográficos (alteração } \\
\text { de metadados e/ou } \\
\text { metainformações) }\end{array}$ \\
\hline
\end{tabular}




\section{ARTIGo}

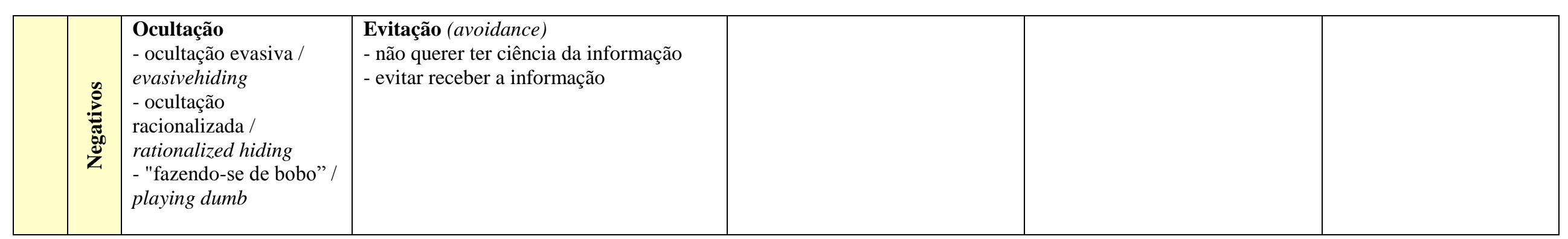

Fonte: Elaboração dos autores 
Nos comportamentos informacionais associados à interação tecnológica o destaque é a tecnologia da informação, com a aplicação de alguns recursos de disponibilização, deixando que os interessados venham buscar o conteúdo necessário (puxar) e outros de transmissão de conteúdo aos interessados (empurrar). Entre as tecnologias de empurrar identificaram-se: email (TURNER, 2012; RAFAELI, RAVID, 2003), transmissão de arquivo (MADLBERGER, 2009), bem como algumas formas mais específicas, tais como a transmissão de arquivos no formato electronic data interchange (EDI) (FUQIANG, 2006). Entre as tecnologias de puxar identificaram-se: blogs, jornais (TURNER, 2012), download de arquivo (KWOK; YANG, 2004) e compartilhamento de bases de dados (MADLBERGER, 2009; BUNTING, LAZENBATT, WALLACE, 2010). Observou-se, também, o comportamento que integra os dois contextos: empurrar somente o índice ou descritivo necessário para que o leitor seja informado do novo conteúdo e possa, posteriormente, fazer o ato de puxar (estratégia puxarempurrar). Neste sentido, Jamali e Nicholas (2008) declaram o comportamento de envio de emails apenas com o índice de conteúdo das novas informações disponíveis no repositório.

Connelly et al. (2012) pesquisaram o não compartilhamento intencional, ou seja, os comportamentos de ocultação da informação. Três comportamentos foram identificados: o de ser evasivo e não atender a solicitação de informação dos colegas de trabalho, denominado de ocultação evasiva (evasive hiding); oferecer uma justificativa para não fornecer a informação solicitada, culpando a outro por esta impossibilidade, comportamento denominado de ocultação racionalizada (rationalized hiding); e fazer-se de ignorante com relação à informação solicitada, não provendo nenhuma informação útil em resposta à solicitação, comportamento denominado de "fazendo-se de bobo" (playing dumb).

As análises resultaram na identificação de quatro comportamentos centrais para compartilhamento da informação: transmissão, disponibilização, verbalização e gesticulação. Há uma ampla possibilidade de variações destes comportamentos que pode ocorrer em função de escolhas associadas às dimensões. Uma das dimensões identificadas é quanto à estruturação da comunicação, se a comunicação é estruturada ou não-estruturada, por exemplo, transmissão de arquivo EDI versus transmissão de e-mail. Outra dimensão é quanto ao emprego da tecnologia, se a comunicação é assistida pela tecnologia ou totalmente humana, como no caso de acesso à base de dados ou fala cara a cara. Outra dimensão é quanto a quantidade de pessoas envolvidas na comunicação, se apenas duas ou em grupo.

\subsection{Sobre comportamento de busca pela informação}

Um aspecto comportamental bastante central no comportamento de busca é quanto à intencionalidade da pessoa em realizar a pesquisa, ou seja, se é uma procura ativa ou passiva. $\mathrm{Na}$ situação ativa a procura por informação é deliberada e intencional (VONTHADEN, 2008), podendo ser inclusive contínua e persistente, ao se configurar um serviço de pesquisa e alerta via e-mail (JAMALI; NICHOLAS, 2008). Na procura passiva, também conhecida como atenção passiva, a aquisição de informação ocorre sem a intenção, por exemplo, assistindo televisão ou andando pelo chão de fábrica (AL-MUOMEN; MORRIS; MAYNARD, 2012). A navegação descomprometida em ambientes virtuais e habituais de 
pesquisa, pré-selecionados, recebe muitas denominações como: casual scanning (VONTHADEN, 2008) e broad scanning (HEINSTROEM, 2006). Já o encontro de informação útil em locais incomuns ou improváveis, é designado de monitoramento não direcionado (nondirected monitoring) (HIRVONEN et al., 2012).

Alguns autores destacam a diferença de comportamento no processo de busca por informação em função da fonte em questão. De Run e Hassan (2003) destacam que as pessoas apresentam maior confiança nas fontes conhecidas e pessoais do que em fontes desconhecidas. Hirvonen et al. (2012) denominam de "active seeking" quando a busca ocorre junto à fonte conhecida e de "active scanning" quando a procura ocorre em fonte desconhecida, esta última envolvendo uma pesquisa de reconhecimento, não tão estruturada e sistemática quanto a que ocorre na "active seeking". Johnson (2007) identificou que o processo de escolha das fontes é dependente do capital intelectual, mais especificamente do capital relacional que abrange a rede social de quem pesquisa.

Há comportamentos informacionais associados aos procedimentos operacionais adotados para condução de busca por conteúdo. Spink (2004) destacou a condução de múltiplas pesquisas em paralelo (multitasking), com o usuário pensando e trabalhando em vários problemas de informação ao mesmo tempo. Kwok e Yang (2004) destacaram a utilização de operadores booleanos para composição de comandos de pesquisa (query) mais eficazes, bem como a forma de considerar e utilizar o indicador de relevância para seleção de conteúdos resultantes da pesquisa. Quanto à categorização de documentos segundo análise de relevância, feita por quem conduz a pesquisa, Makri e Warwick (2010) denominaram-na de comportamento de diferenciação ou distinguishing. Outro comportamento, ligeiramente distinto é quando se considera para seleção de conteúdo o nível de relevância pré-indicado pelo software de busca e a utilidade percebida pelo usuário que realiza a busca, ao qual Makri e Warwick (2010) denominaram de assessing. Weaver et al. (2010) abordaram o comportamento informacional em termos do tempo despendido com atividades de busca. Dobrowolski et al. (2004) destacaram a ação de visitar rapidamente um ambiente virtual de conteúdo e passar rapidamente para outro, ou seja, uma pesquisa rápida (horizontal), sem a realização de uma pesquisa em profundidade (vertical) em nenhum desses ambientes, ação conhecida como boucing ou flicking. O comportamento de planejar o início da pesquisa com um tema amplo e, posteriormente, refinar as pesquisas (vertical) sucessivamente conforme os conteúdos vão sendo encontrados, é denominado de berrypicking (WU, 2011). Ao comportamento de esforço mínimo de busca e seleção, com opção pelo conteúdo cujo acesso seja o mais fácil e rápido, Heinstroem (2006) denominou de fast surfing. A opção contrária, de esforços consideráveis na busca e seleção do conteúdo de alto valor e o mais apropriado possível, Heinstroem (2006) denominou de "mergulho profundo" (deep diving).

As motivações para iniciar a pesquisa também são objeto de estudos associados ao comportamento informacional de busca. Nicholas et al. (2009) destacaram a motivação gerada a partir de uma citação lida, que desperta o interesse do leitor em acessar e ler o texto original que fora citado. Algo muito similar foi indicado por Jamali e Nicholas (2008): a motivação oriunda do comportamento de trilhar documentos descritos em listas de 
referências. VonThaden (2008) destacou como motivação a procura por tendências e a motivada por comunicação informal em que ocorre a recomendação. Nesta mesma perspectiva, Hirvonen et al. (2012) destacaram a procura motivada por indicação de alguma pessoa, como um membro da família ou um profissional da área médica, enquanto Jamali e Nicholas (2008) destacaram os diálogos interpessoais ocorridos em congressos. Thani e Hashim (2011) identificaram o objetivo de ter compreensão inicial de um tema, ou seja, tornar-se mais próximo do assunto, por intermédio de uma exploração inicial. A esta ação de obter um overview do tema, Heinstroem (2006) denominou de exploração ampla. De forma contrária a todos os comportamentos relatados até aqui, Hirvonen et al. (2012) identificaram o comportamento informacional de evitar e adiar a aquisição de informação, em função de ser, muito provavelmente, negativa e indesejável.

As análises do comportamento de busca resultaram na identificação de três comportamentos centrais de busca de informação: pesquisa ativa, observação passiva e evitação. Há uma ampla possibilidade de variações destes comportamentos que pode ocorrer em função de escolhas associadas a dimensões descritas a seguir. A dimensão fonte indica a preferência do pesquisador quanto à fonte, se conhecida ou desconhecida. A dimensão motivação para realização da pesquisa envolve várias razões: rastreamento de uma citação lida e que se deseja acessar e ler o texto original, identificação de documentos descritos em listas de referências, procura por tendências, recomendação de leitura de amigos, indicações comentadas em congressos. Outra dimensão refere-se à lógica de exploração do tema: visitar rapidamente um ambiente virtual de conteúdo e passar rapidamente para outro, ou seja, pesquisas rápidas (horizontal), passando por diversos ambientes, ação conhecida como boucing ou flicking; na situação oposta, ocorre o "mergulho profundo" (deep diving) em um ambiente, ou seja, realiza-se uma pesquisa em profundidade (vertical). Quanto à quantidade de temas pesquisados simultaneamente: condução de múltiplas pesquisas em paralelo (multitasking). Quanto à consideração da relevância de conteúdo no processo de seleção e escolha: utilização do indicador de relevância provido pelo próprio repositório a se realizar a pesquisa (assessing) ou categorização de relevância segundo os critérios definidos pelo pesquisador (distinguishing). Quanto à precisão dos procedimentos de pesquisa: utilização de operadores booleanos para composição de comandos de pesquisa (query) mais precisos ou a situação contrária, do fast surfing, com esforço mínimo de busca e seleção, com o propósito de realização de uma pesquisa de forma mais fácil e rápida.

\subsection{Sobre comportamento de uso da informação}

Segundo Bukowitz e Williams (1999) o uso da informação no contexto das organizações se caracteriza pela aplicação da informação, que se buscou e se obteve, frente a uma situação, a uma demanda específica de sua função profissional. Nos textos pesquisados diversos comportamentos foram identificados como associados ao uso da informação, conforme descritos nos parágrafos a seguir.

A alteração do conteúdo em si foi identificada por alguns pesquisadores como comportamento de uso: Makri e Warwick (2010) consideraram a alteração de imagens e 
vídeos realizada por estudantes de arquitetura como comportamento de uso da informação, assim como Choo et al. (2008) destacaram a re-formatação de conteúdos já existentes. Estas ações são exemplos de comportamentos de uso declarados por Kirk (2002) como "information packaging".

Há comportamentos informacionais identificados como de uso da informação associados à menção ou citação de conteúdo. Frishammar (2003) e Perkins e Rao (1990) indicaram este comportamento quando ocorre a menção do conteúdo para justificar uma tomada de decisão, caracterizando um comportamento de uso denominado por Kirk (2002) como "shaping judgments and decisions". Qi e Ochs (2009) observaram o comportamento de uso quando da citação da informação para elaboração e declaração de uma inferência, comportamento de uso denominado por Kirk (2002) como "developing new knowledge and insights". Nesta mesma categoria se enquadra a citação a conteúdo em mensagens redigidas e postadas em fóruns virtuais direcionadas a agentes financeiros (O'CONNOR, 2013).

Outra forma identificada como evidência do comportamento de uso da informação compreende a mudança de comportamento das ações de tripulações de uma empresa aérea (VONTHADEN, 2008) a partir de informação transmitida. Neste caso observa-se a evidência do comportamento de uso definida por Kirk (2002) como "influencing others".

Alguns pesquisadores consideram apenas o acesso ao conteúdo como suficientes para evidenciar o comportamento de uso: Farag e Lyons (2010) consideraram o acesso a conteúdos sobre o sistema de transporte público pelos usuários deste sistema; Nicholas et al. (2009) consideraram o acesso a páginas de repositórios de biblioteca digital como comportamento de uso da informação por estudantes. Makri e Warwick (2010) consideraram o comportamento de uso indo um pouco além do acesso, incluindo o ato de salvar em meios próprios de armazenamento o arquivo digital do documento ou imagem. Alguns autores associam o comportamento de uso da informação a ações de marcas ou evidências de leitura deixada na mídia que expressa e apresenta o conteúdo como: incluir marcadores (bookmark) na versão digital da informação, comportamento observado por Makri e Warwick (2010) junto a estudantes de arquitetura manuseando conteúdos na forma de imagem e vídeo; inclusão de anotações em folhas impressas de artigos, comportamento observado por Niu e Hemminger (2012) que pesquisaram o comportamento de uso da informação por cientistas.

A presente pesquisa identificou cinco tipos de comportamento informacional de uso considerados pelos pesquisadores: empacotamento da informação (information packaging, de Kirk (2002)), desenvolvimento de novos conhecimentos e insights (developing new knowledge and insights, de Kirk (2002)), direcionamento de julgamentos e decisões (shaping judgments and decisions, de Kirk (2002)), influenciando outros (influencing others, de Kirk (2002)) e reconhecimento da existência da informação. Este último mais associado à leitura e ciência da informação, não implicando necessariamente em aplicação ou uso, porém foi apontado e rotulado como comportamento de uso por alguns pesquisadores. Em relação aos cinco comportamentos informacionais de uso definidos por Kirk (2002), apenas um não foi identificado no levantamento e análise desta pesquisa: o fluxo de informação (information 
flow). Uma nova categoria foi identificada além das cinco definidas por Kirk (2002): reconhecimento da existência da informação.

\subsection{Sobre comportamento de aprendizagem a partir do uso da informação}

Solomon (1997a, 1997b) explora os comportamentos informacionais dos participantes envolvidos na elaboração do plano anual de trabalho de uma agência pública. Ele identificou a aprendizagem a partir de comportamentos associados ao processo de criação de sentido coletivo (sense making) a partir da análise de dados previamente coletados para este fim. Em Solomon (1997c), diversos comportamentos informacionais intrínsecos ao processo de sense making são discutidos como favoráveis à aprendizagem: consistentemente organizar as informações em listas, tabelas e gráficos; desenvolver e utilizar indicadores da empresa para identificar problemas e soluções possíveis; apreciar feedback, mesmo quando negativo.

Apenas outros três artigos identificam comportamentos informacionais associados à aprendizagem. Allen, Karanasios e Slavova (2011) discutem a teoria da atividade aplicada ao ensino e aprendizagem. Entre os comportamentos informacionais associados à aprendizagem destaca-se a contextualização de informações disponíveis. $O$ comportamento de contextualização é descrito como parte do processamento de insumos (informações) do sistema humano voltado à interpretação e construção de significado. Genuis (2012) discute como as mulheres processam informações para aprenderem sobre os efeitos da menopausa. Os comportamentos informacionais associados à aprendizagem são: compilação e comparação de informações provenientes de diferentes fontes. Makri e Warwick (2010) ao relatarem os comportamentos informacionais dos estudantes de arquitetura, descrevem a aprendizagem pela composição, pela criação de pensamentos, ocorridos pelo ato colaborativo da escrita em rede, denominada de "assembling".

Os poucos artigos que abordaram comportamentos informacionais associados à aprendizagem podem ser resumidos em comportamentos de: criação coletiva de sentido (sense making), co-autoria (assembling) e síntese.

\subsection{Sobre comportamento de obtenção / organização da informação}

Spink e Currier (2006) pesquisaram o comportamento informacional de pessoas conhecidas. Entre as categorias de comportamento, definiu-se uma como sendo de organização da informação, destacando comportamentos como: classificação da informação por intermédio de catálogos e criação de índices. Niu e Hemminger (2012) estudaram o comportamento informacional de cientistas e trabalharam com uma categoria denominada coleta de informação, definindo como comportamento desta categoria: a manutenção de base de dados bibliográficos. Desta forma, os poucos comportamentos informacionais de obtenção da informação identificados foram: criação de catálogos de informação, indexação da informação e manutenção de base de dados bibliográficos. 
Para cada um dos 133 artigos da amostra registrou-se a diversidade de comportamentos informacionais abordados pela pesquisa. $\mathrm{O}$ número máximo para o contador de diversidade era cinco, considerando-se o total de unidades de registro utilizadas para análise de conteúdo: "comportamento de compartilhamento", "comportamento de busca", "comportamento de utilização", "comportamento de aprendizagem" e "comportamento de obtenção". Para fins de contagem, consideraram-se os comportamentos informacionais: a) efetivamente analisados qualitativamente ou quantitativamente, por exemplo, em estudos de casos ou experimentos; b) bem como, os comportamentos informacionais apenas descritos e conceituados, abrangendo, por exemplo, os estudos teóricos. Das 133 pesquisas analisadas, 26 não abordavam nenhum comportamento informacional, seja considerando-o como objeto de análise qualitativa ou quantitativa, ou apenas conceituando-o. Isso ocorreu, principalmente, entre os artigos identificados na pesquisa inicial (scanning) pelo uso da palavra-chave "information behavior", muito mais abrangente e genérica que as demais ("information sharing / share", "information use / usage / using" e "information seeking").

Dos 95 artigos que descreveram pelo menos um dos cinco comportamentos informacionais considerados (compartilhamento, busca, utilização, aprendizagem e obtenção), observou-se: que $86(90,6 \%)$ deles descreveram apenas um comportamento informacional, que $6(6,3 \%)$ descreveram dois comportamentos informacionais, e que apenas $3(3,1 \%)$ descreveram três comportamentos informacionais. Os 9 artigos que abordaram mais de um comportamento informacional estão descritos na Quadro 3. Nenhum dos artigos da amostra abordou quatro ou cinco dos comportamentos informacionais considerados nas atividades de análise de conteúdo. Isto demonstra a carência de pesquisas científicas que analisem os comportamentos informacionais de forma diversificada e integrada, segundo a demanda do processo de gestão da informação, processo crítico às KIF.

QUADRO 3 - Artigos que abordaram mais de um comportamento informacional

\begin{tabular}{|c|c|c|c|c|c|c|}
\hline \multirow{2}{*}{ Artigo } & \multirow{2}{*}{$\begin{array}{c}\text { Total de } \\
\text { Comport. }\end{array}$} & \multicolumn{5}{|c|}{ Comportamento Informacional Abordado } \\
\hline & & Compartilhar & Buscar & Utilizar & Aprender & Obter \\
\hline Meyer (2003) & 2 & $\mathrm{X}$ & & $\mathrm{x}$ & & \\
\hline Jamali e Nicholas (2008) & 2 & $\mathrm{X}$ & $\mathrm{x}$ & & & \\
\hline Kwok e Yang (2004) & 2 & $\mathrm{X}$ & $\mathrm{x}$ & & & \\
\hline VonThaden (2008) & 2 & & $\mathrm{x}$ & $\mathrm{x}$ & & \\
\hline Hirvonen et al. (2012) & 2 & & $\mathrm{x}$ & $\mathrm{x}$ & & \\
\hline O'connor (2013) & 2 & $\mathrm{X}$ & & $\mathrm{x}$ & & \\
\hline Spink e Cole (2006) & 3 & & $\mathrm{x}$ & $\mathrm{X}$ & & $\mathrm{x}$ \\
\hline Makri e Warwick (2010) & 3 & & $\mathrm{x}$ & $\mathrm{x}$ & $\mathrm{x}$ & \\
\hline Niu e Hemminger (2012) & 3 & & $\mathrm{x}$ & $\mathrm{x}$ & & $\mathrm{x}$ \\
\hline
\end{tabular}

Fonte: Elaboração dos autores 


\section{CONCLUSÕES}

Para elaboração das conclusões, retorna-se ao objetivo proposto para esta pesquisa: analisar as pesquisas científicas com foco em comportamento informacional, descritas na forma de artigos, verificando a diversidade, conceituação e integração de comportamentos associados às demandas do processo de gestão da informação no contexto das organizações.

Quanto à diversidade de comportamento informacional, observa-se um forte predomínio de pesquisas que discutem a atividade de busca da informação. Dos 157 artigos que retornaram do EBSCO, $92(56 \%)$ se referiam ao comportamento de "information seeking"; enquanto que no ProQuest, observou-se 31 (35\%) de um total de 89 artigos, conforme destacado na Quadro 2. O foco nesta atividade se explica pela análise da dinâmica do ambiente da biblioteca: a) não se atribui aos bibliotecários a responsabilidade pela aprendizagem ou criação de novos conteúdos, o conteúdo já é obtido em sua forma final; b) há pouco foco no compartilhamento, se o leitor guardará para si as informações ou se irá compartilhar com outrem, não se constitui uma preocupação da área; c) não há descarte de informação, no máximo substituição, isso no caso de mídia física (livros deteriorados); d) a obtenção resume-se a solicitar compra e indexar os exemplares recém-adquiridos. Em suma, o desafio atual das KIF, de ter uma dinâmica de transferência da informação entre os indivíduos da(s) organização(ões), trabalhando o conteúdo tácito e explícito, com o objetivo de gerar novos conteúdos a partir dos atuais, requer uma dinâmica e um modelo de comportamento informacional bastante distinto do verificado no contexto das bibliotecas.

Quanto ao vínculo e integração dos diversos comportamentos informacionais para um mesmo indivíduo como, por exemplo, os requeridos ao IW ou $\mathrm{KW}$ em termos de busca, seleção, obtenção para, posterior, aprendizagem, criação (este peculiar apenas ao KW) e compartilhamento, observa-se que: as pesquisas não apresentam esta perspectiva ampla e integradora de comportamentos informacionais que dão suporte às demandas das atividades do processo de gestão da informação do IW ou ao processo de gestão do conhecimento do KW. Conforme destacado na Quadro 3, apenas 9 dos 133 artigos da amostra abordaram mais de um comportamento, sendo 6 artigos abordando dois comportamentos e 3 artigos abordando três comportamentos. Os estudos de comportamento informacional evidenciaramse estanques, muito pontuais, em termos de atividades do processo de gestão da informação ou de gestão do conhecimento. Estes estão distantes da prática e do comportamento requerido do KW que atua nas KIF. Esta segunda constatação acaba sendo coerente com a primeira, ou seja, pouca diversidade de comportamentos abordados para poucas atividades consideradas.

Aos pesquisadores da área de gestão da informação nas organizações evidencia-se a necessidade de se buscar ou desenvolver novos modelos de comportamento informacional, considerando que os atualmente empregados foram concebidos para a realidade da ciência da informação, mais especificamente para a realidade específica e pontual das bibliotecas e a operação dos repositórios digitais. A preocupação histórica deste grupo, para os quais estes modelos foram desenvolvidos, é prioritariamente para com a atividade de busca pela 
informação realizada pelos seus usuários, ou seja, como esses operam os instrumentos disponíveis para encontrar a informação no acervo (CASE, 2002).

A presente pesquisa coopera com as pesquisas científicas na área de gestão da informação nas organizações, ao identificar e descrever subgrupos específicos de comportamentos informacionais associados a cinco comportamentos centrais ao processo de gestão da informação nas organizações: busca, obtenção, uso, aprendizagem e compartilhamento. Essas informações, resumidas na Quadro 1, são íntegras e atuais, considerando-se suas fontes: artigos publicados recentemente em revistas científicas de alto impacto. Entende-se que este conjunto de informações configura-se como um recurso importante a pesquisadores e praticantes da área de gestão da informação nas organizações, por descrever comportamentos que podem ser insumos para futuros modelos teóricos ou para o desenvolvimento e aplicação de abordagens empresariais voltadas à análise do comportamento informacional dos KW.

Como limitação da presente pesquisa, observa-se que algumas das atividades do processo de gestão da informação nas organizações não foram contempladas em termos de comportamentos informacionais, conforme as pesquisas realizadas junto aos dois repositórios de artigos científicos. Este é o caso das atividades de: descarte da informação, identificação da necessidade de informação e análise da informação disponível. Estes aspectos, além de limitações, apresentam-se como sugestão para ampliação e continuidade desta pesquisa.

\section{REFERÊNCIAS}

AL-MUOMEN, N.; MORRIS, A.; MAYNARD, S. Modelling information-seeking behaviour of graduate students at Kuwait. Journal of Documentation, v. 68, n. 4, p. 430-459, 2012.

ALLEN, D.; KARANASIOS, S.; SLAVOVA, M. Working with activity theory: Context, technology, and information behavior. Journal of The American Society For Information Science \& Technology, v. 62, n. 4, p. 776-788, 2011.

ALVESSON, M. Social Identity and the Problem of Loyalty in Knowledge Intensive Companies. Journal of Management Studies, v. 37, n. 8, p. 1101-1123, 2000.

ALVESSON, M. De-Essentializing the Knowledge Intensive Firm: Reflections on Sceptical Research Going against the Mainstream. Journal of Management Studies, v. 48, n. 7, p. 1640-1661, 2011.

BARTLETT, J. C.; TOMS, E. G. Developing a Protocol for Bioinformatics Analysis: An Integrated Information Behavior and Task Analysis Approach. Journal of The American Society For Information Science \& Technology, v. 56, n. 5, p. 469-582, 2005. 
BUKOWITZ, W. R.; WILLIAMS, R. L. The knowledge management fieldbook. London: Pearson Education Limited, 1999.

BUNTING, L.; LAZENBATT, A.; WALLACE, I. Information sharing and reporting systems in the UK and Ireland: Professional barriers to reporting child maltreatment concerns. Child Abuse Review, v. 19, n. 3, p. 187-202, 2010.

CASE, D. O. Looking for Information: A survey of research on information seeking, needs, and behavior. San Diego, CA: Academic Press, 2002.

CHOO, C. W. A organização do conhecimento. 3. ed. São Paulo: Senac, 2011.

$\mathrm{CHOO}, \mathrm{C}$. et al. Information culture and information use: An exploratory study of three organizations. Journal of The American Society for Information Science \& Technology, v. 59, n. 5, p. 792-804, 2008.

CONNELLY, C. E. et al. Knowledge hiding in organizations. Journal of Organizational Behavior, v. 33, n. 1, p. 64-88, 2012.

DE RUN, E. C.; HASSAN, S. H. Evaluation of information sources: a study of Sarawakian business-to-business managers. Journal of Asian Pacific Marketing, v. 2, n. 2, p. 56-72, 2003 .

DOBROWOLSKI, T. et al. Re-appraising information seeking behaviour in a digital environment: Bouncers, checkers, returnees and the like. Journal of Documentation, v. 60, n. 1, p. 24-43, 2004.

DOLAN, T. Revisiting Adhocracy: From rhetorical revisionism to smart mobs. Journal of Future Studies, v. 15, n. 2, p. 33-50, 2010.

FARAG, S.; LYONS, G. Explaining public transport information use when a car is available: Attitude theory empirically investigated. Transportation, v. 37, n. 6, p. 897-913, 2010.

FRENKEL, S. et al. Re-Constituting Work: Trends Towards Knowledge Work and InfoNormative Control. Work, Employment and Society, v. 9, n. 4, p. 773-796, 1995.

FRISHAMMAR, J. Information use in strategic decision making. Management Decision, v. 41, n. 4, p. 318-326, 2003.

FUQIANG, Z. Competition, Cooperation, and Information Sharing in a Two-Echelon Assembly System. Manufacturing \& Service Operations Management, v. 8, n. 3, p. 273 291, 2006. 
GENUIS, S. K. Constructing "sense" from evolving health information: A qualitative investigation of information seeking and sense making across sources. Journal of the American Society for Information Science and Technology, v. 63, n. 8, p. 1553, 2012.

GOREY, R. M.; DOBAT, D. R. Managing in the Knowledge Era. The Systems Thinker, Waltham, v. 7, n. 8, p. 1-5, 1996.

HASSAN IBRAHIM, N.; ALLEN, D. Information sharing and trust during major incidents: Findings from the oil industry. Journal of The American Society For Information Science \& Technology, v. 63, n. 10, p. 1916-1928, 2012.

HEINSTROEM, J. Broad exploration or precise specificity: Two basic information seeking patterns among students. Journal of the American Society for Information Science and Technology, v. 57, n. 11, p. 1440-1450, 2006.

HIRVONEN, N. et al. Information behavior in stages of exercise behavior change. Journal of The American Society for Information Science \& Technology, v. 63, n. 9, p. 18041819, 2012.

HISLOP, D. Conceptualizing Knowledge Work Utilizing Skill and Knowledge-based Concepts: The Case of Some Consultants and Service Engineers. Management Learning, v. 39, n. 5, p. 579-596, 2008.

JAMALI, H. R.; NICHOLAS, D. Information-seeking behaviour of physicists and astronomers. Aslib Proceedings, v. 60, n. 5, p. 444-462, 2008.

JOHNSON, C. A. Social capital and the search for information: Examining the role of social capital in information seeking behavior in Mongolia. Journal of The American Society For Information Science \& Technology, v. 58, n. 6, p. 883-894, 2007.

KARUNAKARAN, A.; SPENCE, P. R.; REDDY, M. C. Towards a model of collaborative information behavior. In: FOSTER, J. (Ed.) Collaborative information behavior: User engagement and communication sharing. Hershey, PA: Information Science Reference, 2010. p.1-5.

KIRK, J. Theorising Information Use: Managers and Their Work. 2002. 345p. PhD Thesis (Doctor Phylosophy) - Faculty of Humanities and Social Sciences - University of Technology Sydney, Sydney, 2002.

KWOK, S. H.; YANG, C. C. Searching the peer-to-peer networks: The community and their queries. Journal of the American Society for Information Science and Technology, v. 55, n. 9, p. 783-793, 2004. 
LILJENQUIST, K. A. ; GALINSKY, A. D.; KRAY, L. J. Exploring the rabbit hole of possibilities by myself or with my group: The benefits and liabilities of activating counterfactual mind-sets for information sharing and group coordination. Journal of Behavioral Decision Making, v. 17, n. 4, p. 263-279, 2004.

LYTRAS, M. D.; POULOUDI, A. Project management as a knowledge management primer: The learning infrastructure in knowledge-intensive organizations. The Learning Organization, v. 10, n. 4/5, p. 237-250, 2003.

MADLBERGER, M. What drives firms to engage in interorganizational information sharing in supply chain? International Journal of e-Collaboration, v. 5, n. 2, p. 18-42, 2009.

MAKRI, S.; WARWICK, C. Information for inspiration: Understanding architects' information seeking and use behaviors to inform design. Journal of The American Society For Information Science \& Technology, v. 61, n. 9, p. 1745-1770, 2010.

MEYER, H. J. Information use in rural development. New Review of Information Behaviour Research, v. 4, n. 1, p. 109-125, 2003.

MISHRA, B.; BHASKAR, A.U. Knowledge management process in two learning organisations. Journal of Knowledge Management, v. 15, n. 2, p. 344-359, 2011.

NICHOLAS, D. et al. Student digital information-seeking behaviour in context. Journal of Documentation, v. 65, n. 1, p.106-132, 2009.

NIU, X.; HEMMINGER, B. M. A study of factors that affect the information-seeking behavior of academic scientists. Journal of The American Society for Information Science \& Technology, v. 63, n. 2, p. 336-353, 2012.

NONAKA, I.; TAKEUCHI, H. The knowledge-creating company: How Japanese companies create the dynamics of innovation. New York: Oxford University Press, 1995.

O'CONNOR, L. G. Investors' information sharing and use in virtual communities. Journal of The American Society for Information Science \& Technology, v. 64, n. 1, p. 36-47, 2013.

PARIDON, T. J. Antecedents in retail information sharing research: The case for personal shopping values and consumer self-confidence. Journal of Applied Management and Entrepreneurship, v. 10, n. 4, p. 18-31, 2005.

PERKINS, W.; RAO, R. C. The Role of Experience in Information Use and Decision Making by Marketing Managers. Journal of Marketing Research, v. 27, n. 1, p. 1-10, 1990. 
QI, L.; OCHS, J. Information use and transference among legally separated share markets--an experimental approach. Southern Economic Journal, v. 76, n. 1, p. 99-129, 2009.

RAFAELI, S.; RAVID, G. Information sharing as enabler for the virtual team: An experimental approach to assessing the role of electronic mail in disintermediation. Information Systems Journal, v. 13, n. 2, p. 191-206, 2003.

SOLOMON, P. Discovering information behavior in sense making. I. Time and timing. Journal of the American Society for Information Science, v. 48, n. 12, p. 1097-1108, 1997a.

SOLOMON, P. Discovering information behavior in sense making. II. The social. Journal of the American Society for Information Science, v. 48, n. 12, p. 1109-1126, 1997b.

SOLOMON, P. Discovering information behavior in sense making. III. The person. Journal of the American Society for Information Science, v. 48, n. 12, p. 1127-1138, 1997c.

SPINK, A. Multitasking information behavior and information task switching: An exploratory study. Journal of Documentation, v. 60, n. 4, p. 336-351, 2004.

SPINK, A.; COLE, C. Human information behavior: Integrating diverse approaches and information use. Journal of The American Society for Information Science \& Technology, v. 57, n. 1, p. 25-35, 2006.

SPINK, A.; CURRIER, J. Toward an evolutionary perspective for human information behavior. Journal of Documentation, v. 62, n. 2, p. 171-193, 2006.

STARBUCK, W. H. Learning by Knowledge-intensive Firms. Journal of Management Studies, v. 29, n. 6, p. 713-40, 1992.

SVEIBY, K. A knowledge-based theory of the firm to guide in strategy formulation. Journal of Intellectual Capital, v. 2, n. 4, p. 344-358, 2001.

THANI, R. A.; HASHIM, L. Information needs and Information seeking behaviors of Social Science Graduate Students in Malaysian Public Universities. International Journal of Business and Social Science, v. 2, n. 4, p. 137-143, 2011.

TURNER, D. Oral documents in concept and in situ, part I: Grounding an exploration of orality and information behavior. Journal of Documentation, v. 68, n. 6, p. 852-863, 2012.

VONTHADEN, T. L. Distributed information behavior: A study of dynamic practice in a safety critical environment. Journal of The American Society for Information Science \& Technology, v. 59, n. 10, p. 1555-1569, 2008. 
WEAVER, J. B. et al. Health information-seeking behaviors, health indicators, and health risks. American Journal of Public Health, v. 100, n. 8, p. 1520-1525, 2010.

WILSON, T. D. Models in information behavior research. Journal of Documentation, v. 55, n. 3, p. 249-270, 1999.

WOFFORD, J. C.; CALABRO, P. J.; SIMS, A. The Relationship of Information Sharing Norms and Leader Behavior. Journal of Management, v. 1, n. 1, p. 15-23, 1975.

WU, I. Toward supporting information-seeking and retrieval activities based on evolving topic-needs. Journal of Documentation, v. 67, n. 3, p. 525-561, 2011.

Como citar este documento:

DE SORDI, José Osvaldo; AZEVEDO, Marcia Carvalho de; MEIRELES, Manuel. Análise Crítica da Pesquisa em Comportamento Informacional Segundo as Demandas da Empresa Intensiva em Conhecimento. Revista Digital de Biblioteconomia e Ciência da Informação, Campinas, SP, v. 13, n. 3, p. 504-525, set. 2015. ISSN 1678-765X. Disponível em: <http://periodicos.sbu.unicamp.br/ojs/ index.php/rdbci/article/view/8635775>. Acesso em: 18 Set. 2015. 